\title{
Large Metastatic Cardiac Tumor from Ascending Colon Cancer with Autopsy
}

\author{
Takashi Tomiyama ${ }^{a}$ Takuya Shijimaya $^{a}$ Yasuki Sano ${ }^{a}$ \\ Sanshiro Kobayashi ${ }^{a}$ Toshiro Fukui ${ }^{a}$ Mitsuaki Ishida ${ }^{b}$ \\ Makoto Naganuma ${ }^{a}$ \\ aDivision of Gastroenterology and Hepatology, Third Department of Internal Medicine, \\ Kansai Medical University, Osaka, Japan; bivision of Diagnostic Pathology, Kansai Medical \\ University, Osaka, Japan
}

\section{Keywords}

Autopsy $\cdot$ Colon cancer $\cdot$ Heart ventricles $\cdot$ Neoplasm metastasis $\cdot$ Palliative care

\begin{abstract}
A metastatic cardiac tumor from colon cancer is an exceedingly rare clinical feature associated with a poor prognosis without therapeutic intervention; however, such cases may be frequently encountered in clinical practice, especially among the elderly. We report a case of synchronous double cancer of the prostate and ascending colon with metastases to multiple organs, including a large cardiac tumor. A 71-year-old Japanese man had prostate cancer with neck and para-aortic metastasis. He visited our hospital with complaints of fatigue and a positive fecal occult blood test result. Colonoscopy findings revealed the presence of a tumor in the ascending colon, and contrast-enhanced CT revealed a tumor in the heart, which was possibly due to metastasis from the ascending colon. The patient received palliative care and declined anticancer treatment. He died due to respiratory failure 3 months after the first diagnosis but did not show critical arrhythmia until death. Autopsy revealed the presence of a large mass in the right ventricle with tumor embolism of the right coronary artery. The cardiac mass was pathologically consistent with metastasis from the colon. In case of colorectal cancer with cardiac metastasis involving poor prognosis or performance status, best supportive care without any therapeutic intervention could be the optimal treatment for the quality of the remaining time.
\end{abstract}

\section{Karger"}


Tomiyama et al.: Metastatic Cardiac Tumor

\section{Introduction}

Colorectal cancer (CRC) is the most common cancer and the second most common cause of cancer-related death in Japan. Even after surgical resection, approximately $20 \%$ of patients with CRC develop distant metastasis, often resulting in a rapidly fatal course. While the liver and lungs are the most common sites of metastases in CRC, metastatic cardiac tumors are extremely rare $[1,2]$. CRC and prostate cancer are the third and second most common malignant tumors, respectively, worldwide [3]. Although metastatic cardiac tumors from colon cancer are a rare clinical feature, such cases may be encountered in daily clinical situations among the elderly. Herein, we report our experience and autopsy findings in a case of ascending colon cancer with an exceptionally large metastatic cardiac tumor that led to respiratory arrest and death.

\section{Case Presentation}

A 71-year-old Japanese man diagnosed with prostate cancer with neck and para-aortic metastasis was referred to our department because of fatigue and a positive fecal occult blood test result. The patient had a 2-year history of chemotherapy using bicalutamide, degarelix, leuprorelin, and docetaxel for prostate cancer. Physical examination revealed a mildly tender right abdominal mass. Laboratory findings showed a white blood cell count of 17,300 cells $/ \mu \mathrm{L}$ and C-reactive protein level of $4.2 \mathrm{mg} / \mathrm{dL}$. Colonoscopy revealed the presence of a circumferential type 2 tumor measuring $50 \times 30 \mathrm{~mm}$ in the ascending colon causing 50\% stricture (Fig. 1a). Pathological findings of the biopsy specimens demonstrated moderate-to-poorly differentiated adenocarcinoma (Fig. 1b). Contrast-enhanced CT showed a $100 \times 70 \mathrm{~mm}$ metastatic tumor at the apex of the heart (Fig. 2a) and multiple metastases to the liver, spleen (Fig. 2b), and lungs (Fig. 2c). Echocardiography results indicated attachment of the metastatic mass to the right ventricular wall of the heart (Fig. $2 \mathrm{~d}$, e). While serum carcinoembryonic antigen levels were found to be elevated at $15.3 \mathrm{ng} / \mathrm{mL}$ (normal range, 0-5 ng/mL), prostate-specific antigen levels were within the normal range. Thus, he was diagnosed with ascending colon cancer with metastases to the heart, liver, and spleen. The patient declined anticancer treatment and opted for best supportive care after a discussion with the clinician and his family. Signs of critical arrhythmia were not detected during the clinical course of the disease. The patient ultimately died due to heart failure 3 months after diagnosis. Autopsy was performed after reaching an agreement with the family. Gross examination showed that the metastatic tumor occupied almost the entire right ventricular cavity (Fig. 3a, b). Pericardial effusion (200 mL) was noted, but tumor invasion on the exterior of the heart was not observed. Histological examination of the cardiac tumor confirmed the diagnosis of metastasis from ascending colon cancer (Fig. 3c). Tumor embolism was observed in the right coronary artery. The cause of death was determined to be respiratory failure due to loss of cardiac output.

\section{Discussion}

The incidence of cardiac metastasis is low but nonignorable in the clinical course of malignancies. In fact, a previous literature review reported that the incidence of cardiac metastasis was $1.23 \%$ in all autopsy cases [4]. Furthermore, cardiac metastasis accounts for $1 \%$ of metastases from colorectal and prostate cancer [5]. One limitation encountered in this case was that the patient declined cancer therapy, and our patient died from heart failure

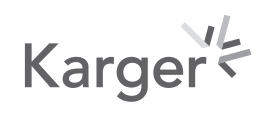



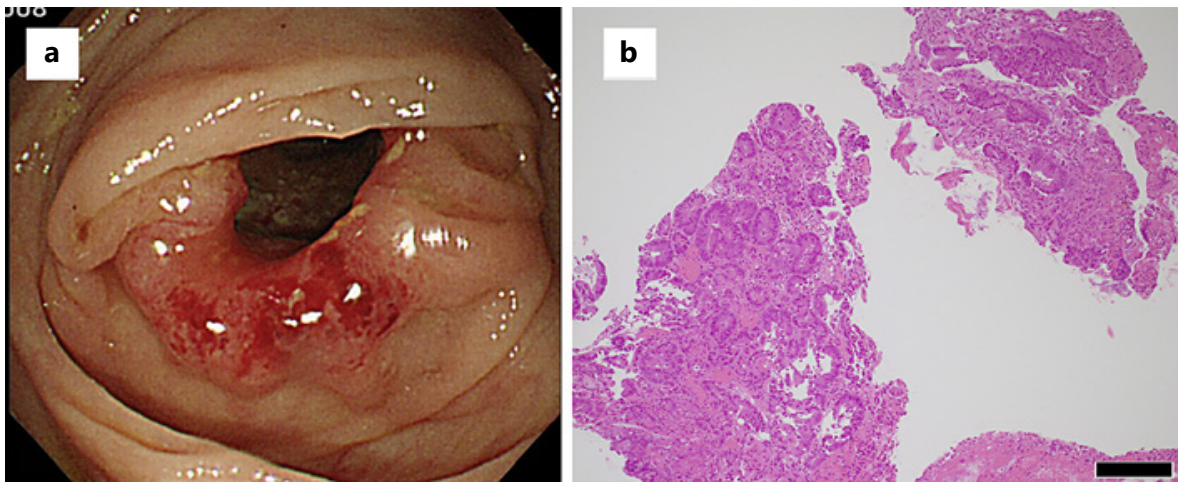

Fig. 1. Endoscopic findings. a Colonoscopy revealed a 50-mm type 2 tumor in the ascending colon. b Hematoxylin and eosin staining of the biopsy specimen revealed moderate-to-poorly differentiated adenocarcinoma. Black bar indicates $200 \mu \mathrm{m}$.
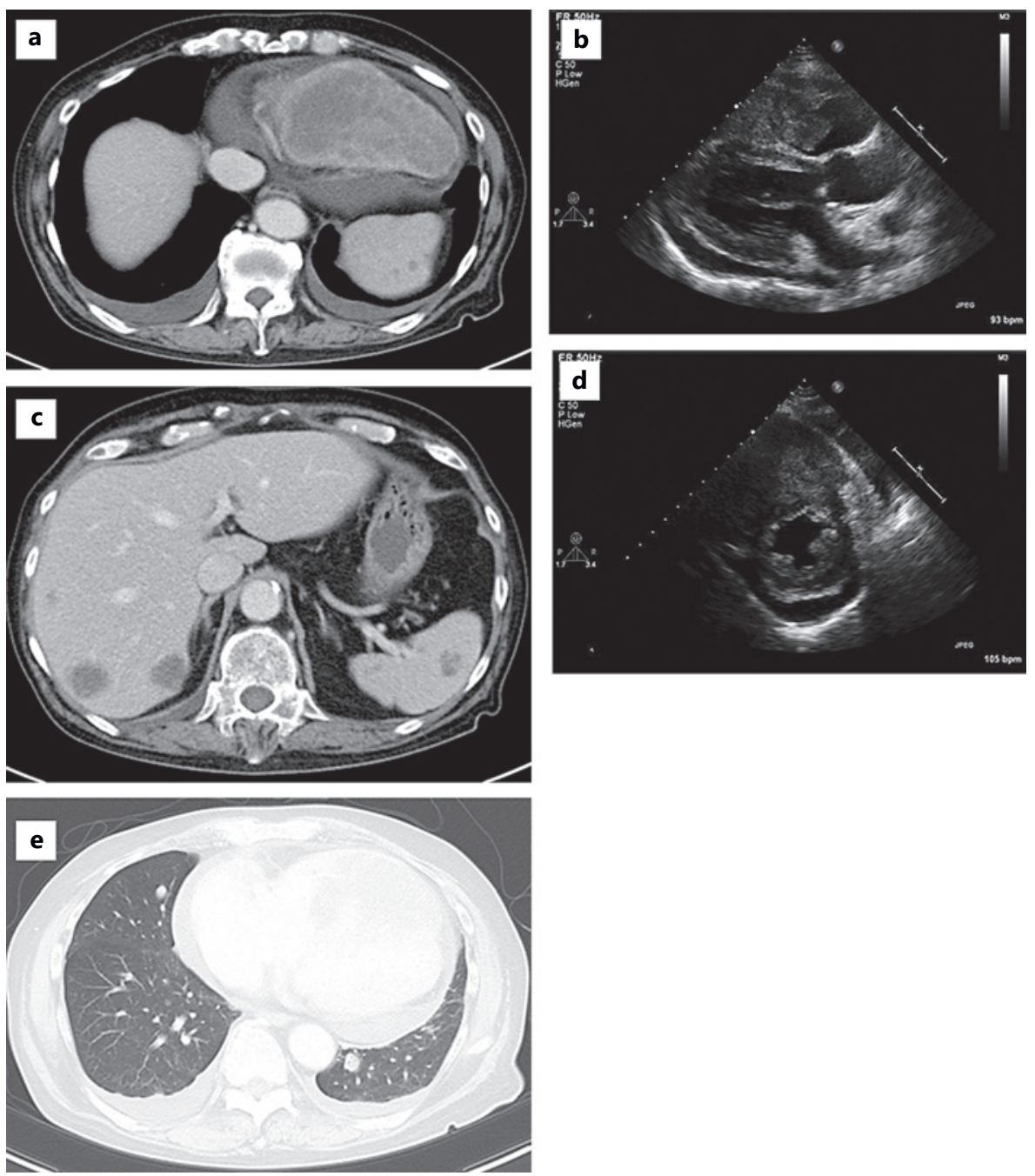

Fig. 2. Findings of contrast-enhanced CT and echocardiography. Systemic contrast-enhanced CT revealed multiple organ metastases: a large mass with a diameter of $100 \times 70 \mathrm{~mm}$ at the apex of the heart (a), liver and spleen metastases (b), and lung metastasis (c). Parasternal long- (d) and short-axis (e) views showing a large tumor mass in the right ventricular wall of the heart. 
Fig. 3. Autopsy findings. a A metastatic cardiac tumor was excised from the right ventricular wall of the heart. b Pathologically, the cardiac tumor was determined to be an adenocarcinoma, consistent with the diagnosis of metastasis from ascending colon cancer. H-E-stained section. Black bar indicates $100 \mu \mathrm{m}$. c Tumor thrombosis was observed in the right coronary artery. H-E-stained section. Black bar indicates $500 \mu \mathrm{m}$.
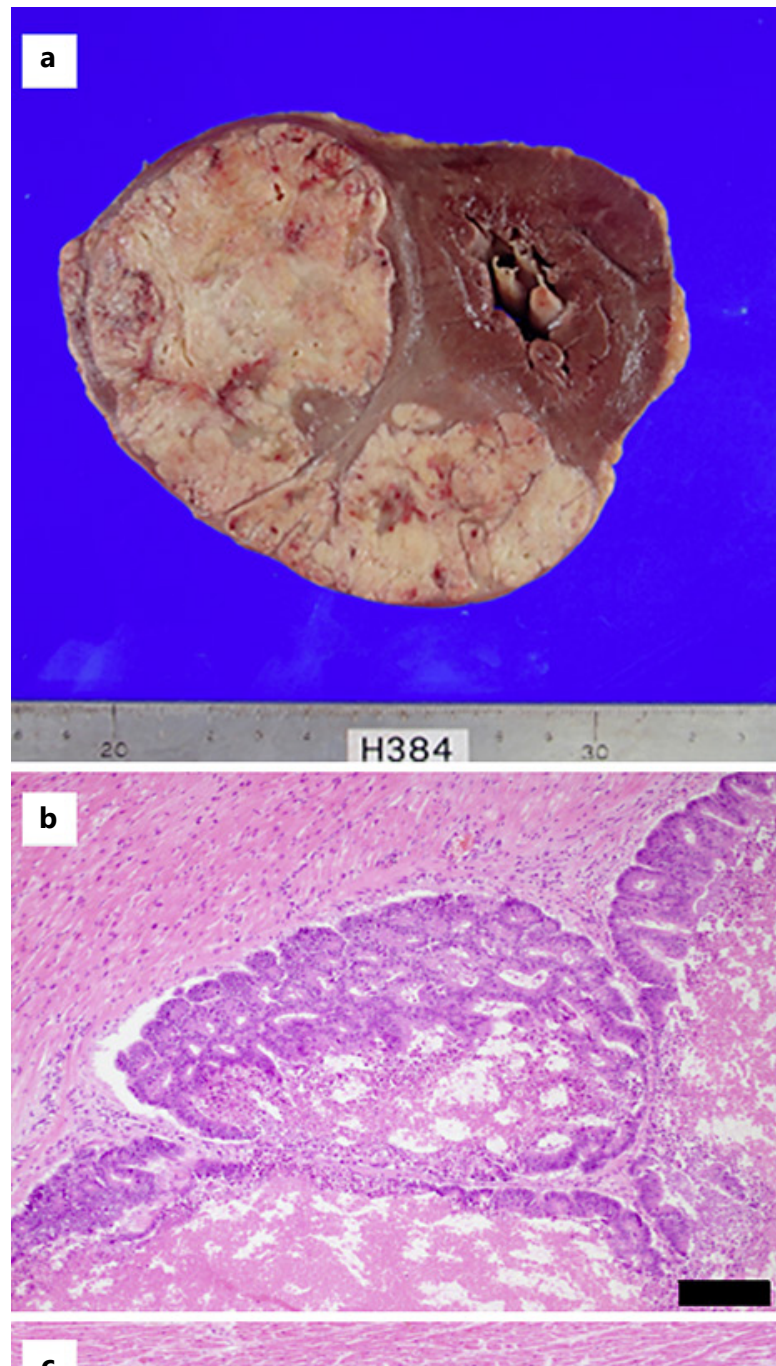

c

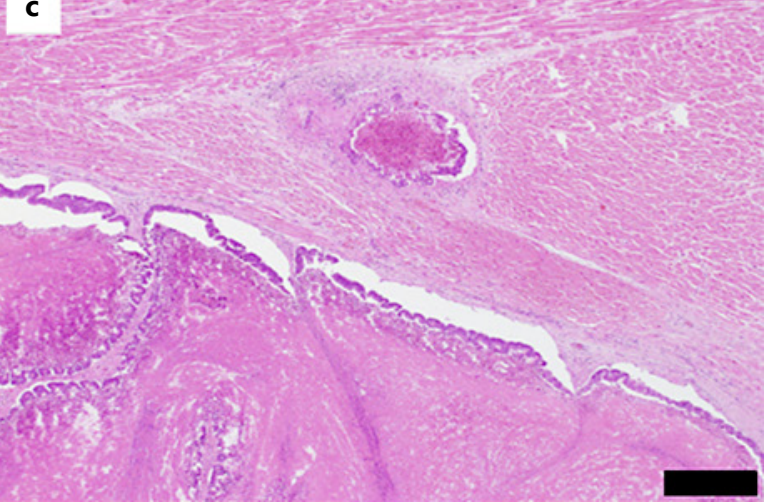

without critical arrhythmia event. Best supportive care without therapeutic intervention could therefore be the optimal approach if the case was "fatalist," similar to our case.

Cardiac tumor symptoms include arrhythmia, thrombosis, and heart failure depending on the tumor location and size [6]. Cardiac metastasis is driven through various pathways such as direct invasion, hematogenous and lymphogenous dissemination, and extension into either the inferior vena cava or the pulmonary veins [5]. In our case, autopsy findings showed that the 
mass occupied the myocardia of the right ventricle; tumor cells were also observed in the right coronary artery implying that this metastasis occurred through hematogenous dissemination.

In our case, a previous diagnosis of colorectal and prostate cancer with multiple metastatic sites concealed the presence of the heart tumor. Previous studies suggested that surgical resection of cardiac tumors was safe and effective for palliation [7]. In cases in which the heart is the only site of metastasis, surgery could be a curative intervention. However, patients with an estimated poor prognosis or disseminated lesions may be more suitable for radiation and/ or chemotherapy if found sensitive [8]. In contrast, there are extremely few case reports in which the use of chemotherapy for the treatment of CRC with cardiac metastasis is reported $[9,10]$. Tsujii et al. [10] reported the efficacy of modified FOLFOX (5-fluorouracil, leucovorin, and oxaliplatin) and panitumumab for the treatment of transverse colon cancer with wildtype KRAS and cardiac metastasis, although this efficacy of FOLFOX was not shown in the other report [9]. In our case, the patient was asymptomatic until the end stage when lowoutput heart failure occurred. Hence, in case of CRC with cardiac metastasis associated with poor prognosis or performance status or in case patients decline cancer therapy as seen in our patient, best supportive care without therapeutic intervention could be the optimal approach for maintaining quality of life in the remaining time.

We report a case of an exceptionally large cardiac tumor derived from CRC without any lethal cardiac event. Therapeutic intervention for such a case should be decided upon considering the balance between benefit and risk.

\section{Acknowledgment}

We would like to thank Editage (www.editage.jp) for English language editing.

\section{Statement of Ethics}

This case report was reviewed, and the need for approval was waived by the Ethics Committee of Kansai Medical University. Written informed consent was obtained from the family of the patient for publication of this case report and any corresponding images before performing the autopsy.

\section{Conflict of Interest Statement}

The authors have no conflicts of interest to declare.

\section{Funding Sources}

This manuscript did not receive any funding.

\section{Author Contributions}

T. Tomiyama wrote the draft of the manuscript. T. Shijimaya, Y. Sano, S. Kobayashi, T. Fukui, M. Ishida, and M. Naganuma assisted in the preparation of the manuscript. All authors have critically reviewed the manuscript.

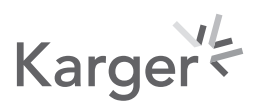




\section{References}

1 Augestad KM, Bakaki PM, Rose J, Crawshaw BP, Lindsetmo RO, Dørum LM, et al. Metastatic spread pattern after curative colorectal cancer surgery. A retrospective, longitudinal analysis. Cancer Epidemiol. 2015 Oct; 39(5):734-44.

2 Riihimäki M, Hemminki A, Sundquist J, Hemminki K. Patterns of metastasis in colon and rectal cancer. Sci Rep. 2016 Jul;6:29765.

3 Bray F, Ferlay J, Soerjomataram I, Siegel RL, Torre LA, Jemal A. Global cancer statistics 2018: GLOBOCAN estimates of incidence and mortality worldwide for 36 cancers in 185 countries. CA Cancer J Clin. 2018 Nov;68(6): 394-424.

4 Lam KY, Dickens P, Chan AC. Tumors of the heart. A 20-year experience with a review of 12,485 consecutive autopsies. Arch Pathol Lab Med. 1993 Oct;117(10):1027-31.

5 Bussani R, De-Giorgio F, Abbate A, Silvestri F. Cardiac metastases. J Clin Pathol. 2007 Jan;60(1):27-34.

6 Hoffmeier A, Sindermann JR, Scheld HH, Martens S. Cardiac tumors--diagnosis and surgical treatment. Dtsch Arztebl Int. 2014 Mar;111(12):205-11.

7 Mkalaluh S, Szczechowicz M, Torabi S, Schmack B, Sabashnikov A, Dib B, et al. Surgical treatment of cardiac tumors: insights from an 18-year single-center analysis. Med Sci Monit. 2017 Dec;23:6201-9.

8 Al-Mamgani A, Baartman L, Baaijens M, de Pree I, Incrocci L, Levendag PC. Cardiac metastases. Int J Clin Oncol. 2008 Aug;13(4):369-72.

9 Pizzicannella J, Ricci V, Gorla R, Spinapolice E, Esposito A. Isolated cardiac metastasis from colorectal cancer in a 35-year-old man. Case Rep Med. 2012;2012:751761.

10 Tsujii Y, Hayashi Y, Maekawa A, Fujinaga T, Nagai K, Yoshii S, et al. Cardiac metastasis from colon cancer effectively treated with 5-fluorouracil, leucovorin, and oxaliplatin (modified FOLFOX6) plus panitumumab: a case report. BMC Cancer. 2017 Feb;17(1):152. 